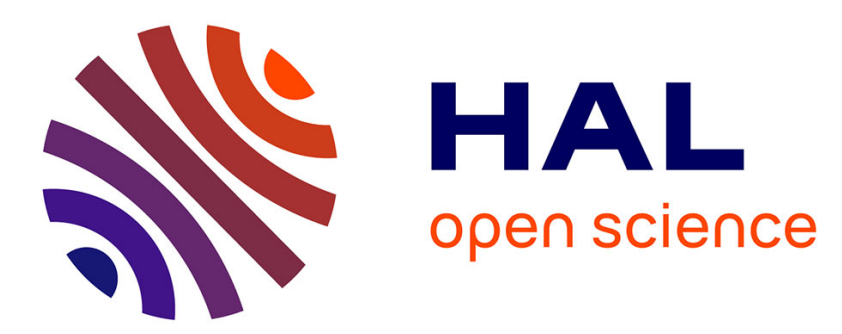

\title{
Numerical investigation of optimal divertor gas baffle closure on TCV
}

D. Galassi, H Reimerdes, C Theiler, M. Wensing, H. Bufferand, G. Ciraolo, P Innocente, Y Marandet, P Tamain

\section{- To cite this version:}

D. Galassi, H Reimerdes, C Theiler, M. Wensing, H. Bufferand, et al.. Numerical investigation of optimal divertor gas baffle closure on TCV. Plasma Physics and Controlled Fusion, 2020, 62 (11), pp.115009. 10.1088/1361-6587/abb24f . hal-03085421

\section{HAL Id: hal-03085421 \\ https://hal.science/hal-03085421}

Submitted on 21 Dec 2020

HAL is a multi-disciplinary open access archive for the deposit and dissemination of scientific research documents, whether they are published or not. The documents may come from teaching and research institutions in France or abroad, or from public or private research centers.
L'archive ouverte pluridisciplinaire HAL, est destinée au dépôt et à la diffusion de documents scientifiques de niveau recherche, publiés ou non, émanant des établissements d'enseignement et de recherche français ou étrangers, des laboratoires publics ou privés. 


\title{
Numerical investigation of optimal divertor gas baffle closure on TCV
}

\author{
D. Galassi ${ }^{1}$, \\ H. Reimerdes ${ }^{1}$, \\ C. Theiler ${ }^{1}$, \\ M. Wensing ${ }^{1}$, \\ H. Bufferand ${ }^{2}$, G. Ciraolo ${ }^{2}$, P. Innocente ${ }^{3}$, Y. Marandet ${ }^{4}$, \\ P. Tamain ${ }^{2}$, the EUROfusion MST1 Team ${ }^{5}$ and the TCV Team ${ }^{6}$
}

1 École Polytechnique Fédérale de Lausanne (EPFL), Swiss Plasma Center (SPC), CH-1015 Lausanne, Switzerland

2 IRFM, CEA Cadarache, F-13108 St. Paul-lez-Durance, France

3 Consorzio RFX, Corso Stati Uniti 4, 35127, Padova, Italy

4 Aix-Marseille Univ., CNRS, PIIM, UMR 7345, Marseille F-13397, France

${ }^{5}$ See the author list of B. Labit et al 2019 Nucl. Fusion 59086020

6 See the author list of S. Coda et al 2019 Nucl. Fusion 59112023

e-mail address: davide.galassi@epfl.ch

\begin{abstract}
A first set of divertor gas baffles has recently been installed in the TCV tokamak. In order to explore the physics determining the benefits and limitations of divertor baffling and to guide the design of a possible second generation of baffles, the effect of baffle closure is investigated using the 2D transport code SolEdge2DEIRENE with realistic wall geometries. The baffle extension is scanned, first imposing the same upstream conditions as in previous SOLPS-ITER studies, then extending the parameter space to access detached plasma conditions. In attached plasma cases, divertor neutral compression is maximised by a LowField Side baffle length with an opening between the separatrix and the baffle tip of approximately $5 \lambda_{q}$, resulting in an increase in compression by a factor 4 with respect to the unbaffled case. In detached cases this ratio can be improved by up to a factor 25 using higher baffle closures. This difference in behaviour between attached and detached conditions is explained by a model based on the ionisation mean free path of neutral particles recycled from the target. In some conditions, the optimal baffle extension in terms of neutral compression is found to be subject to high levels of intercepted upstream heat flux, which results in a peak heat flux on the baffles comparable to the one impinging on the outer
\end{abstract}


target. The individual roles of the High-Field Side and Low-Field Side baffles are disentangled by means of dedicated simulations, which show a lower global impact of the inner baffle. This study suggests that an outer baffle with a gap of approximately $3 \lambda_{q}$, slightly longer than the one presently installed, could further enhance the neutral compression ratio in cases where the ionisation front is detached. The biggest unknown in these simulations is related to far SOL particle transport, which could result in higher levels of baffle recycling and thus limit baffle performance.

\section{Introduction}

Divertor baffling and the resulting improvement of the confinement of neutral particles in the divertor region of tokamaks is known to facilitate the access to detached divertor conditions (see e.g. [1]). Several baffling solutions have been conceived (see e.g. [2]), including advanced solutions such as a tight baffling along divertor legs [3], baffles enclosing the target region in a Super-X divertor [4], small-angle slot divertors [5] and V-shaped divertors [6]. Even though the baffle geometry can change according to specific tokamaks, several physical problems are common to all of them, such as the maximisation of the neutral confinement in the divertor, the interplay with plasma plugging and the requirement to assure acceptable heat and particle fluxes impinging on the baffles. In this work, we numerically study gas baffling and its limitations for the specific solution currently implemented on the Tokamak à Configuration Variable (TCV) [7], which consists in a narrow throat at a height close to the X-point. The main goal of this study is to understand the effects that determine the optimal baffle design for different divertor conditions.

The TCV baffle is formed by an inner baffle, located on the High-Field Side (HFS) of the vessel at a vertical location of about $Z=-0.3 \mathrm{~m}$ with respect to the midplane, and by an outer baffle located at the Low-Field Side (LFS) at a similar height, as visible in figure $1([8],[9])$. A unique characteristic of the TCV baffles is their capacity to largely preserve the flexibility of the divertor magnetic geometry. The vertical positioning of the baffles, relatively far from the bottom where the outer strike point usually sits, is compatible with multiple magnetic geometries, including Lower Single Null, X, Super-X and Snowflake divertors [9]. As a result of the baffles, an increased density of neutral atoms and molecules recycled from the wall is expected in the divertor volume, along with a decrease in neutral density at upstream locations. The increased neutral density in the divertor should enhance momentum and power losses from the plasma, facilitating the access to the detached regime and extending the operational range of dissipative divertor operation in $\mathrm{L}$ and $\mathrm{H}$-mode plasmas on TCV. 
The dimensions of the first generation of baffles, recently installed in TCV, were designed ([10]) to maximise the neutral compression ratio:

$$
c_{D} \equiv n_{n}^{\text {div }} / n_{n}^{\text {main }},
$$

where $n_{n}=n_{D^{0}}+2 n_{D_{2}}$ is the neutral density, and the superscripts div and main indicate the divertor and the main chamber regions, respectively. The design is based on edge plasma simulations performed with the code SOLPS-ITER ([11],[12]). In this code, the fluid solver B2 is coupled with EIRENE [13], a Monte-Carlo code which solves a kinetic model for neutrals, allowing the description of plasma-neutrals and plasmawall interactions. A current limitation of SOLPS-ITER is that the plasma solver grid is not allowed to intercept solid objects other than the target plates, and in particular it is limited in radial extent by the position of the baffle tips. As a consequence, sheath boundary conditions are only applied at the target plates. Plasma flux radially out of the simulation domain is dependent on ad-hoc boundary conditions, e.g. imposed on the radial density decay length, which can become a sensitive input parameter if the simulation domain is limited in radial extent. Therefore, the plasma flux impinging on gas baffles cannot be modelled self-consistently.

SOLPS simulations carried out in [10] suggested that the largest neutral compression would be obtained with a baffle placed at a distance from the separatrix corresponding to approximately 5 characteristic heat flux widths $\lambda_{q}$. For longer baffles (Baffles 4 and 5 in [10]), however, the mesh grid had to be shrunk in the radial direction, making the results more sensitive to the boundary conditions imposed at the outer boundary of the plasma grid. On the contrary, in cases where the grid did not extend up to the baffle tip, an artificial channel was possibly left open for neutrals to leak from the divertor to the main SOL, yet without any plasma transport into the divertor. These limitations and the sensitivity of the recycling at the outer boundary of the plasma grid on ad-hoc boundary conditions warrant further studies.

In the present paper, we investigate in more detail the optimal choice of the baffle length and the plasma-baffle interaction with the SolEdge2D-EIRENE code [14], which allows for a plasma grid extension and realistic boundary conditions up to the first wall and the baffles. The aim is to better understand the physics mechanisms determining the benefits and limitations of divertor baffling, to complement experimental findings from the first baffled campaign on TCV and guide the design for a second set of baffles. The paper is organised as follows. In section 2, the SolEdge2D-EIRENE code and simulation setups will be described. In section 3 we present a scan in outer baffle length, fixing upstream plasma conditions. The individual roles of the inner and the outer baffle will be assessed for the same input parameters, by carrying out simulations with each of these components alone. This analysis is useful as well since some restrictions given by the TCV baffles on magnetic geometry could be relaxed if just one of the baffles, the HFS or the LFS one, is proven to be sufficiently effective. In 
section 4 we extend the outer baffle scan to different upstream conditions, in particular to higher upstream density and lower power, and we discuss the variation of the neutral compression ratio in all the simulated cases. In section 5 we will present a simple model useful to understand the impact of the baffles on the achieved neutral compression ratio in different divertor plasma conditions. Conclusions are presented in section 6 .

\section{SolEdge2D-EIRENE simulations setup}

SolEdge2D is a 2D multi-fluid code solving Braginskii equations, with a diffusive ansatz on cross-field transport. Being coupled with EIRENE, it also includes plasma-neutrals interactions. In this code, the above-mentioned limitation on the plasma mesh grid is overcome by means of a numerical technique called "penalisation" [15]. In SolEdge2DEIRENE, the grid of the plasma solver and the one of EIRENE are superimposed. A mask function determines if a grid cell belongs to the wall or to the vacuum. In case of a wall cell, an additional term is solved in the equations, that forces the evolved quantity to a certain value. One can demonstrate [15] that, with an adequate choice of the penalisation parameters, this technique allows to recover the Bohm boundary conditions at the interface between plasma and plasma-facing components. Figure 1 shows an example of the parallel Mach number $M$ distribution over the plasma wall interface in SolEdge2D-EIRENE simulations, confirming that the Mach values in the first cell included in the wall are always $|M| \geq 1$.

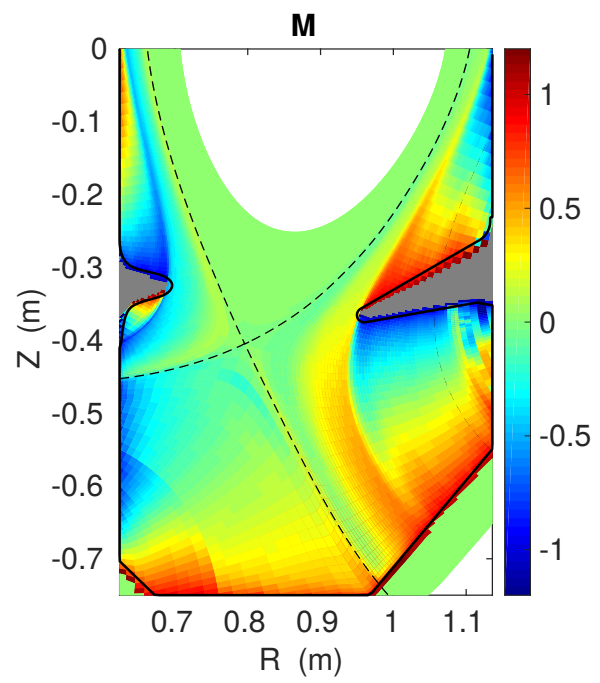

Figure 1: Example of parallel Mach number distribution in the SolEdge2D domain for a baffled TCV plasma. The baffle tiles are coloured in grey.

In this work, we simulate the divertor performance of a standard TCV single-null 
magnetic configuration that had already been used in previous studies [10], with several shapes for the baffle geometry, as illustrated in figure 2. The Baffle 1 considered in [10] has been omitted, since it was found to be far from the optimum design.

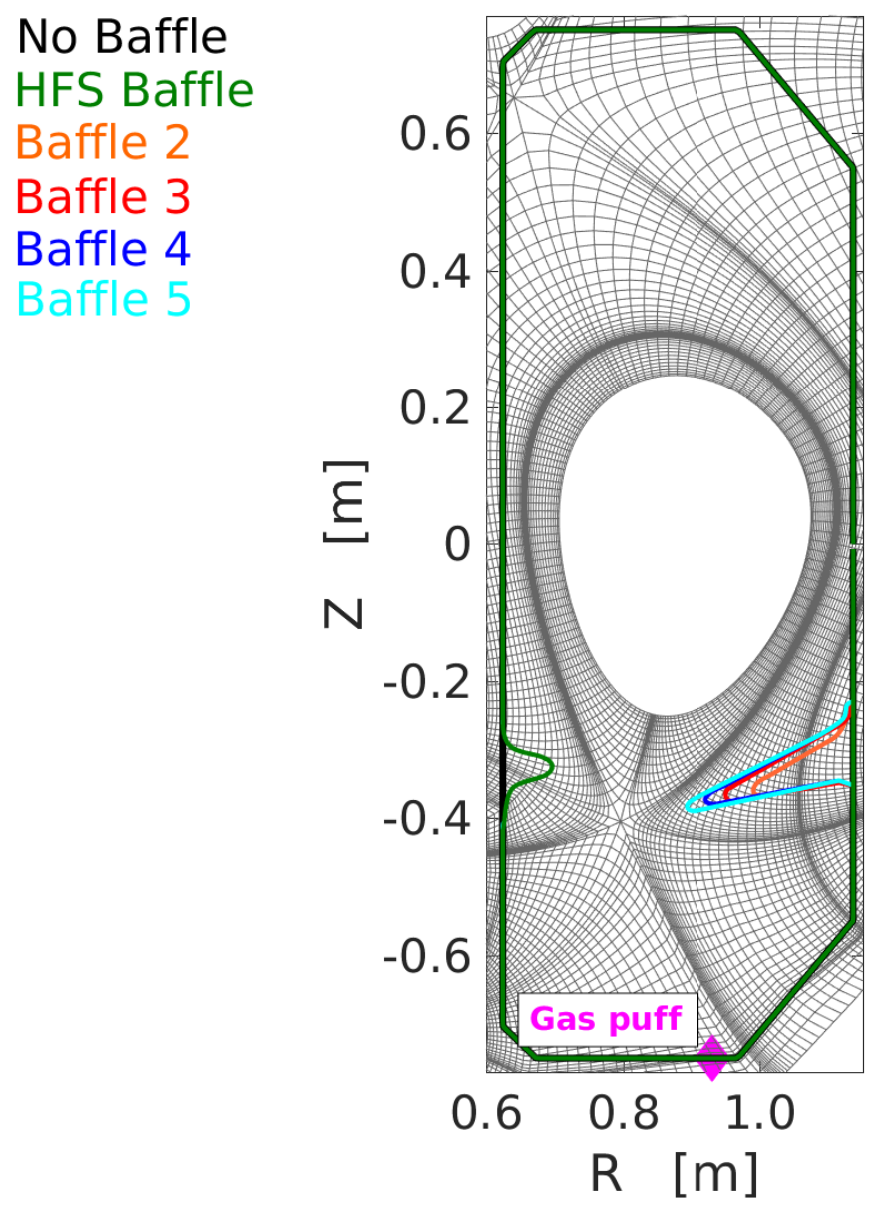

Figure 2: Simulated wall geometry in the different baffle cases. The geometry of the inner baffle is the same in all cases.

The design of the first version of the baffles installed on TCV is similar to Baffle 3 , shown in figure 2. The X-point is centered between the baffles, whose tips are tangent to the same flux surface. In order to determine the distance across flux surfaces, we use the coordinate $\rho=\sqrt{\psi-\psi_{0}} / \sqrt{\psi_{x 1}-\psi_{0}}$, where $\psi_{0}$ is the poloidal flux at the magnetic axis, and $\psi_{x 1}$ is the one at the separatrix. Table 1 lists the distance of the baffle tip from the separatrix for each considered case.

The distance of the baffle from the separatrix is also measured in SOL heat flux 


\begin{tabular}{l|c|c} 
& $\rho_{b}$ & $\Delta \rho / \lambda_{q}$ \\
\hline No Baffle & 1.083 & 5.9 \\
HFS Baffle & 1.057 & 4.1 \\
LFS Baffle 2 & 1.120 & 8.6 \\
LFS Baffle 3 & 1.069 & 4.9 \\
LFS Baffle 4 & 1.043 & 3.1 \\
LFS Baffle 5 & 1.025 & 1.8
\end{tabular}

Table 1: Normalised flux coordinate of the closest point of the baffle (or of the outer wall in the No Baffle case), with respect to the separatrix, and distance from the separatrix of the same point, measured in $\lambda_{q}$.

widths $\lambda_{q}$. This is also a useful parameter to characterise the baffle extension, since the optimal neutral compression must be determined assuring a sustainable heat flux on the baffles. At the LFS baffle poloidal location $\lambda_{q} \simeq 4.3 \mathrm{~mm}$, corresponding to a $\Delta \rho \sim 0.014$. This value depends on the transport coefficients imposed in the simulations, and it varies by less than $10 \%$ percent among all simulations, when transport coefficients are kept constant.

The SolEdge2D grid is flux-surface aligned. The resolution of the grid in the poloidal direction is of the order of $1 \mathrm{~cm}$ at locations far from the strike points, while it is refined to $1 \mathrm{~mm}$ near the strike points and of $5 \mathrm{~mm}$ close to the baffle tips, where large gradients are usually expected. The radial resolution at the outer midplane is of the order of $1 \mathrm{~mm}$ near the separatrix, and increases further out. Transport coefficients are imposed to be constant in time and in the radial direction. In the poloidal direction, they follow a Gaussian profile centered on the midplane, in order to mimic a ballooning-like turbulent transport. The poloidal average of the transport coefficients for particles, electron heat flux and ion heat flux, of $D_{\perp}=0.2 \mathrm{~m}^{2} / \mathrm{s}, \chi_{\perp}^{e}=1.0 \mathrm{~m}^{2} / \mathrm{s}$ and $\chi_{\perp}^{i}=1.0 \mathrm{~m}^{2} / \mathrm{s}$ correspond to the values chosen in the SOLPS investigation [10] to match heat flux experimental profiles at the outer target in a particular non-baffled TCV L-mode discharge (\#52312). Similarly, drifts are not taken into account in this work.

Deuterium molecules are puffed at the location shown in figure 2, which corresponds to the location of the standard fuelling valve in TCV. A feedback loop adjusts the simulated gas puff to control the electron density at the outboard midplane separatrix. The actual density can differ up to $\sim 3 \%$ with respect to the target value. The deuterium recycling coefficient on the carbon wall is set to $R=0.986$, which results in a relatively good agreement between gas puff levels in the simulations and in the experiment. The simulations include carbon ionised states, where the only source of carbon atoms are physical and chemical sputtering. The physical sputtering is calculated with the Bohdansky formula [16], while the chemical sputtering yield is set to 
$3.5 \%$, as in [10]. The imposed value for the carbon recycling coefficient on carbon tiles is 0.4: since this coefficient is hard to be actually determined from experiments, the value has been chosen in order to obtain a reasonable radiated power in the SOL. Only the continuity and the momentum conservation equations are solved for carbon ions, which are considered in thermal equilibrium with the main ions.

The upstream conditions imposed in the simulations discussed in this paper are summarised in table 2 . The LFS baffle 4 in table 2 indicates the geometry, that will be discussed in section 3.3, including only the outer baffle of Baffle 4 .

\begin{tabular}{l|c|c} 
& $n_{e, u}\left(10^{19} \mathrm{~m}^{-3}\right)$ & $P_{i n}(M W)$ \\
\hline No Baffle & $1.8 ; 3.5$ & $0.3 ; 1.2$ \\
HFS baffle & 1.8 & 1.2 \\
Baffle 2 & $1.8 ; 3.5$ & $0.3 ; 1.2$ \\
Baffle 3 & $1.8 ; 3.5$ & $0.3 ; 1.2$ \\
Baffle 4 & $1.8 ; 3.5$ & $0.3 ; 1.2$ \\
LFS baffle 4 & 1.8 & 1.2 \\
Baffle 5 & $1.8 ; 3.5$ & $0.3 ; 1.2$
\end{tabular}

Table 2: Summary of the electron upstream density and input power imposed in the presented simulations. The density value is imposed at the outer midplane, at the separatrix position.

In high-power simulations, $1 / 3$ of the power is assigned to electrons and $2 / 3$ to ions, as in [10], while in low power simulations it is evenly distributed between the two species.

\section{Baffle closure scan at fixed upstream condition}

In this section, we inspect the effect of different baffle closures on both outer midplane (upstream) and target profiles, fixing $n_{u}=1.8 \cdot 10^{19} \mathrm{~m}^{-3}$ and $P_{\text {in }}=1.2 \mathrm{MW}$. These are the same low-collisionality upstream conditions as in [10].

\subsection{Upstream profiles}

While the upstream electron density at a flux surface just inside the separatrix is fixed, the upstream neutral density varies substatially with the baffle dimensions, as shown in figure 3c. It decreases monotonically with increasing baffle length up to Baffle 4, where a reduction of approximately a factor 10 is achieved. Beyond this point, it increases again in the case of Baffle 5. This behaviour is due to a competition of a better 
neutral confinement in the divertor with increasing baffle length and increasing levels of recycling on the baffle. It should also be noted here that neutrals recycled from the baffles get mostly ionised in the close vicinity of the baffle. Therefore, even more than to the upstream neutral density, they contribute to the average main chamber neutral density, which is the quantity used in section 4 to determine neutral compression.

The neutral particle density at the outer midplane is the main difference with respect to former SOLPS simulations [10], where it increased considerably with respect to the optimum case for Baffles 4 and 5 (by one order of magnitude with Baffle 5). However, these were also the cases for which, in SOLPS, the plasma grid was radially narrowed. As a result, these simulations were particularly sensitive to the choice of the radial outer boundary condition, i.e. the value set for the radial density decay length. Comparison with the present SolEdge2D-EIRENE simulations indicates that the choice of this boundary condition in [10] resulted in an overestimation of the level of main chamber recycling. This effect, together with a different poloidal distribution of main chamber recycling (predominantly from the baffles in SolEdge2D-EIRENE) is likely the cause of the discrepancy in the two simulations.
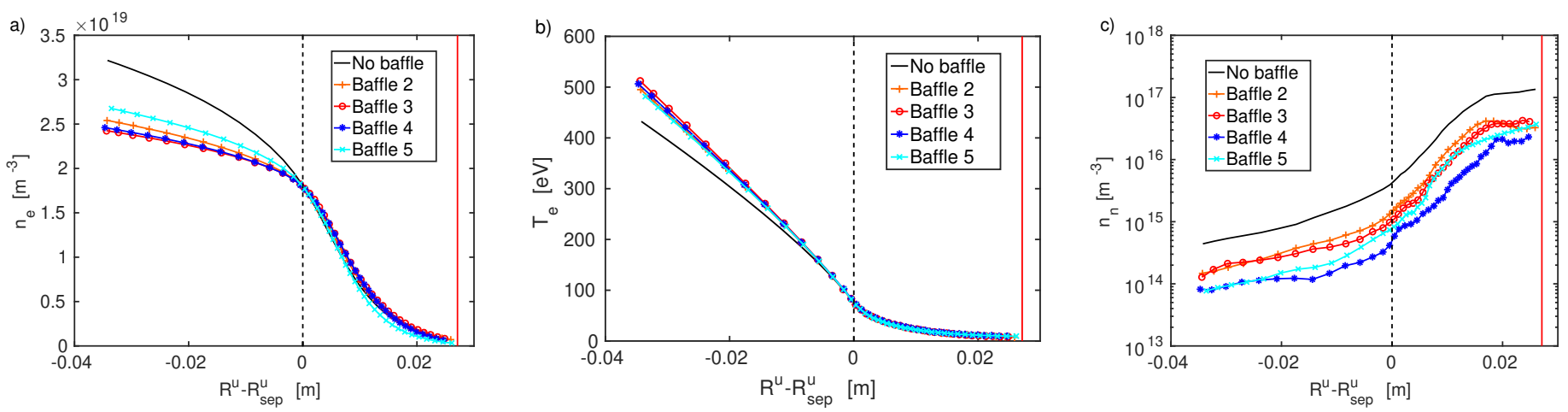

Figure 3: a): Electron density at the outer midplane in the low-collisionality case, in function of the baffle length. The dashed black vertical line represents the separatrix, while the red vertical line represents the first wall. b) Upstream (outer midplane) electron temperature. c) Upstream neutral density $n_{n}=n_{D^{0}}+2 n_{D_{2}}$.

Electron density at the outer midplane changes modestly from case to case, as it is shown in figure 3a. In the first flux surfaces of the SOL, density with the baffles is up to $15 \%$ higher (Baffle 3 case) as compared to the unbaffled case. This is due to the fact that Baffle 3 is the one characterised by the lowest ionisation in the confined region. Therefore, a lower density gradient at the separatrix is necessary to transport particles outwards. Since we fix the electron density just inside the separatrix, a flatter density gradient leads to higher densities in the first SOL flux surfaces. This difference in the steepness of density profile is more visible in the closed flux surfaces. The flattening of the density profile in the confined region has also been observed on 


\begin{tabular}{l|c|c} 
& Gas Puff $\left(10^{21} \mathrm{el} / \mathrm{s}\right)$ & Radiated power $(\mathrm{kW})$ \\
\hline No Baffle & 1.4 & 360 \\
HFS Baffle & 1.6 & 390 \\
Baffle 2 & 1.9 & 400 \\
Baffle 3 & 2.0 & 410 \\
Baffle 4 & 1.9 & 380 \\
LFS baffle 4 & 1.8 & 330 \\
Baffle 5 & 2.1 & 340
\end{tabular}

Table 3: In the second column, values of the gas puff in the different baffle configurations needed to ensure a fixed upstream density. In the third column, the values of the power radiated in the simulation domain.

DIII-D, in experiments where a closed divertor configuration was compared to an open one [17]. The electron and ion temperatures (only shown here for electrons) follow an opposite trend in the confined region with respect to density: this happens because of the fixed power imposed in the simulations, and from the assumption of a diffusive cross-field heat transport. One can also notice from figure $3 \mathrm{~b}$ ) that temperature in the SOL remains unaffected by the baffle dimensions. This is consistent with previous SOLPS-ITER simulations [18].

The gas puff required to keep the upstream density constant increases in the presence of the investigated baffles by $\sim 40 \%$, but depends little on the dimensions of the baffle, Table 3. This can be understood since in the presence of baffles, the divertor volume is better decoupled from the main chamber and thus a higher gas puff is needed to achieve the same separatrix upstream density. Two additional simulations have been performed, featuring the same plasma upstream conditions, although imposing the gas puff at the top of the machine $($ at $(R, Z)=(0.92,0.75) \mathrm{m}$ ). These simulations were once performed without baffle and once with Baffle 3. Also in this case, the simulation with the Baffle 3 results in a higher gas puff than in the unbaffled geometry. However, this increase is lower $(\sim 20 \%)$ than in the case where the gas puff is at the bottom. This is interpreted to be due to the fact that puffed neutrals now reach the closed-field lines region in the same way in baffled and unbaffled cases, while the baffle still partially screens the neutrals recycled at the strike points. The increase in gas puff values in baffled cases has also been observed in simulations with higher upstream density, with gas puffing both from the bottom and from the top of the machine. 


\subsection{Target profiles}

In baffled cases, the electron density at the outer target increases by up to $40 \%$ with Baffle 3, with respect to the simulation with no baffle (figure 4a). It is weakly sensitive to a change in the outer baffle extension and does not show a systematic trend. The peak neutral density at the outer target follows a similar trend as electron density, with a maximum for Baffle 3 (not shown). The electron and ion temperatures at the target (only shown for electrons, in figure 4b) are considerably more strongly affected by the choice of the baffle, decreasing monotonically with the baffle length.
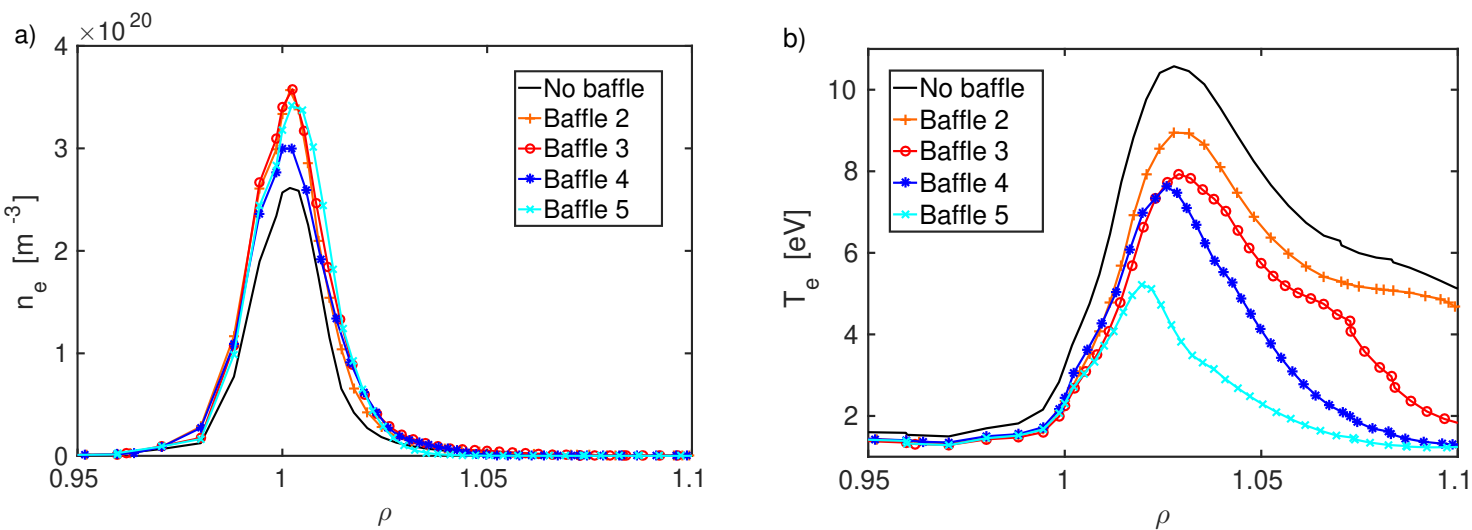

Figure 4: a): Electron density profiles at the outer target in the low-collisionality case, as function of the baffle length. b) Electron temperature at the outer target.

To explain this strong variation in target temperature with the baffles, we show the total heat flux impinging on the outer strike point, superimposed with the one affecting the outer baffle, in figure 5a. This heat flux includes the contributions from ions, electrons and neutrals. At $\rho<1.02$, the heat flux is approximately the same in all cases, meaning that the radiative power losses are not significantly affected by the baffles in these low-collisionality plasmas. Nevertheless, even at these locations the electron temperature in the baffled cases is lower with respect to the unbaffled one. This is due to the fact that the total pressure is approximately conserved on the flux surfaces, so an increase in target density leads to the observed decrease in target temperature.

Even for $\rho>1.02$ the target heat flux is not significantly affected by radiated power levels. Table 3 shows that the total radiated power (which is mainly due to weakly-ionised states of carbon in the relatively cold divertor region) does not change dramatically with different baffle geometries and it is maximised in the Baffle 2 and 3 cases, because of the high values of electron density near the targets. The strong reduction in target temperature with increasing baffle length for $\rho>1.02$ can instead be 

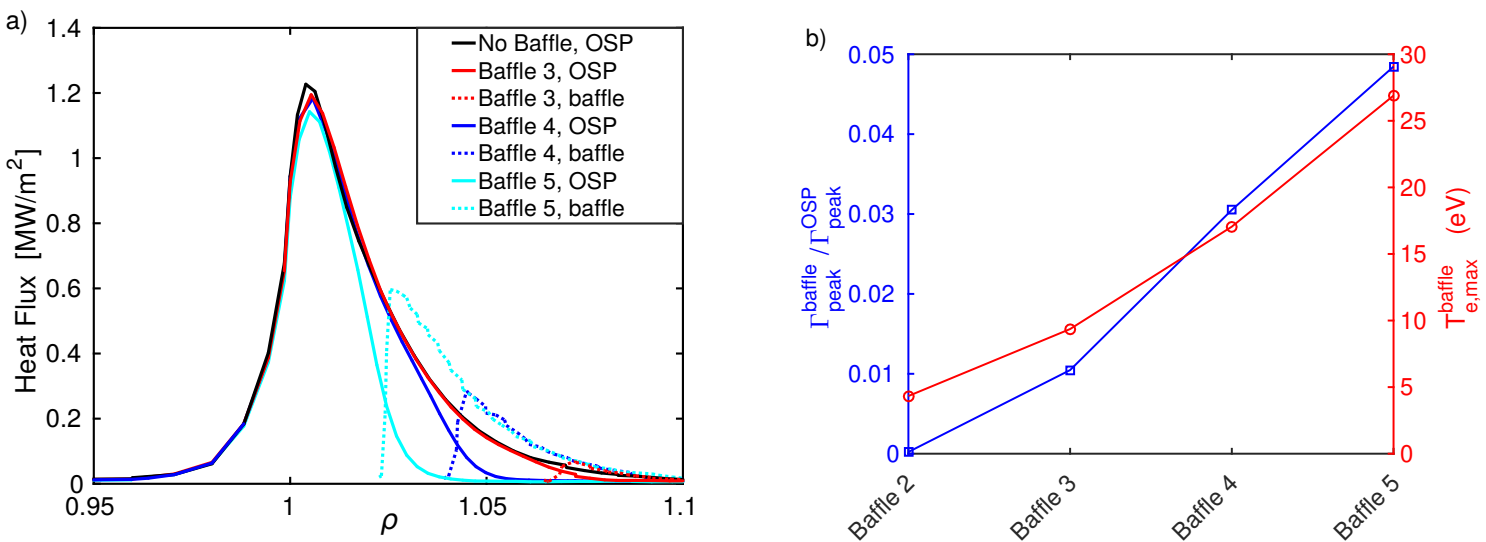

Figure 5: a) Total heat flux projected on the wall surface at the outer strike point, superimposed to the one on the outer baffle, for different baffle lengths. b) Ratio of the peak particle flux $(\Gamma)$ projected on the wall surface at the baffle and at the outer strike point, and maximum temperature at the baffle.

explained by a stronger and stronger interception of the heat flux by the outer baffle, as visible in 5a. Therefore, the longer the baffle, the smaller the integrated heat flux reaching the outer strike point, and the lower the target temperature. In the Baffles 4 and 5 cases, the peak heat flux intercepted by the outer baffle corresponds to $\sim 25 \%$ and $\sim 50 \%$ of the peak heat flux at the target, respectively. Clearly, such high heat flux fractions on the baffles might be a concern in the design of baffle tiles in a reactor. In addition, such baffle interaction can cause enhanced levels of impurity sputtering and resulting impurity fluxes towards the confined plasma. In the present simulations, however, carbon sputtered from the baffles does not penetrate significantly into the closed field-line region.

Besides the parallel heat flux, the parallel particle flux is also affected by the presence of the baffles. However, as visible in figure 5b, the peak ion flux on the baffle tip, even in the Baffle 5 case, is significantly lower than at the strike points. In fact the overall particle source in the flux tubes connected to the baffle is small, if compared to the flux tubes connected to the target, leading to small ion fluxes and elevated temperatures at the baffle surface. Moreover, the smaller connection length between the midplane and the outer baffle, with respect to the one between midplane and target, leads in our simulations to a sheath-limited regime in the low-field side of the flux tubes connected to the baffles, with high peak temperatures (up to $\sim 30 \mathrm{eV}$ ) at the baffle tip, as visible in figure 5b.

The ion flux on the baffles may depend on transport properties. Simulations with $D_{\perp}$ 
increased by a factor 2 and 6 have been run with the same baffle geometries as before to check the sensitivity to these parameters, while the other transport coefficients have been kept constant. Even with these higher values of $D_{\perp}$, and thus broader midplane density profiles, the peak particle flux on the outer baffle has been found to be much lower with respect to the one at the outer target, not changing the trends observed in figure 5. The ion flux is weakly sensitive on $D_{\perp}$ since the majority of the ions stays confined in a recycling pattern around the divertor target, and it is weakly affected by upstram transport conditions. The balance between intercepted fluxes of heat and particles by the baffles, although found to be weakly dependent on the values of $D_{\perp}$ explored here, would probably change for flat far-SOL density profiles and in particular in presence of a "density shoulder" [19]. The result would be a higher recycling on the baffle, and thus higher neutral particle fluxes to the confined region. The study of this problem should be addressed by dedicated edge turbulence simulations.
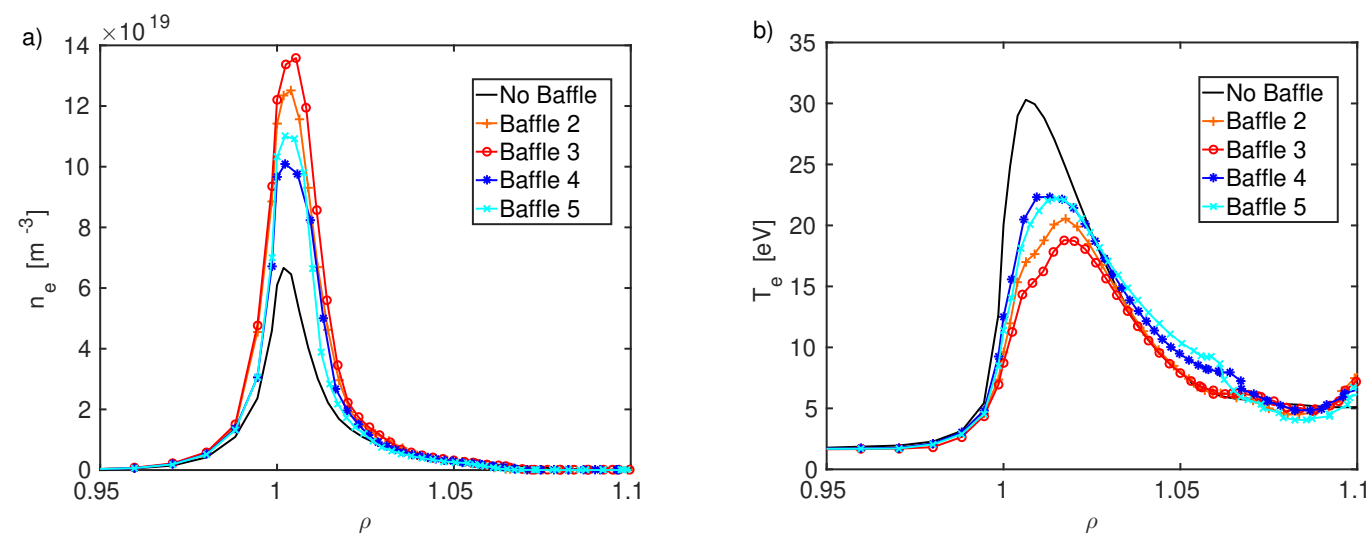

Figure 6: a): Electron density at the inner target in the low-collisionality case, in function of the baffle length. b) Electron temperature at the inner target.

At the inner target the electron density increases in baffled cases, as visible in figure $6 \mathrm{a}$, by a larger factor than at the outer target. The peak density follows the same trend as a function of the baffle length as at the outer target. Electron and ion temperatures are lowered (only shown for $T_{e}$ ) by the presence of the baffle, figure $6 \mathrm{~b}$. This reduction in target temperature is consistent with weak changes in upstream and target pressures for varying baffle length. Contrary to the outer target, there is no substantial interception of parallel heat flux by the inner baffle and consequently no monotonic decrease of the temperature at the inner target with an increasing outer baffle extension. 


\subsection{The effect of the inner and outer baffles}

In order to evaluate the separate effects of the inner and of the outer baffles, we analyse the two simulations where only the HFS baffle, or only the LFS baffle 4 are included. Among the different LFS baffles, we simulate the one corresponding to Baffle 4, since it results, in most of the cases, in the highest neutral compressions.

The inner baffle alone lowers the electron temperature at the inner target by approximately $30 \%$, as visible in figure $7 \mathrm{~b}$. At the outer target, instead, it has a very weak effect on the electron temperature, figure 7a. The neutral compression (see section 4.1 for details) is increased by only $20 \%$ with the inner baffle alone.
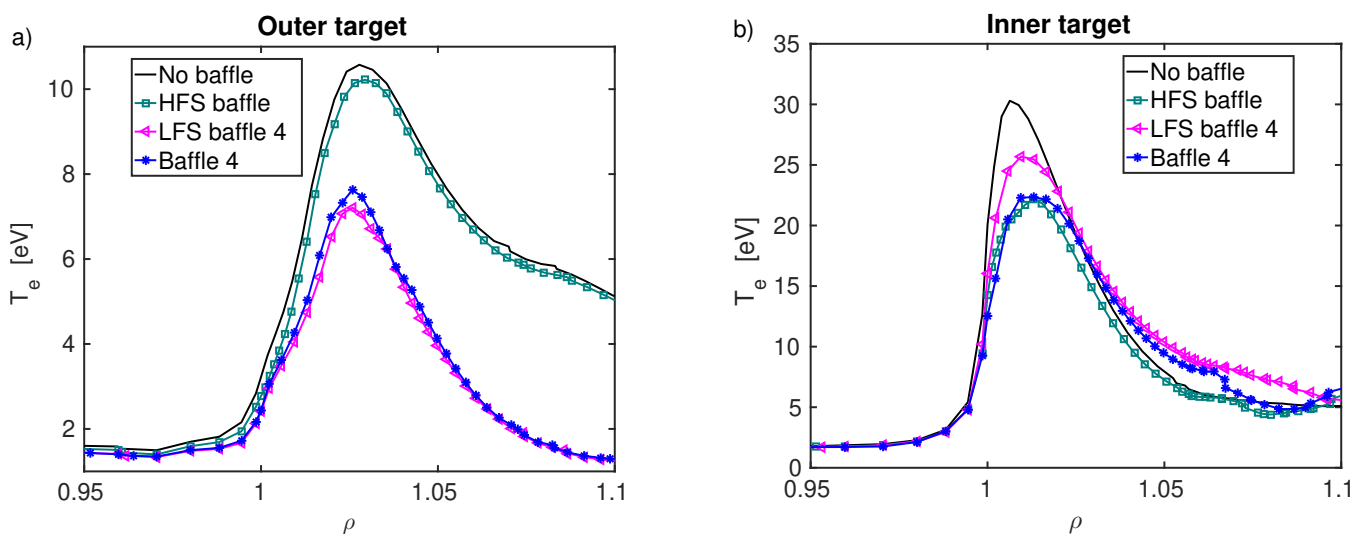

Figure 7: a) Electron temperature at the outer target in the low-collisionality case, for geometries without baffle, with HFS or LFS baffle 4 only, and with the full Baffle 4 . b) Electron temperature at the inner target.

The outer baffle 4 alone, besides cooling down the outer target, also lowers the peak electron temperature at the inner target by approximately $15 \%$, without intercepting directly the heat flux directed to the HFS (figure 7b). At the outer target, the outer baffles leads to plasma conditions very similar to the full Baffle 4. Neutral compression is increased in this case by a factor 3 with respect to the unbaffled case, similarly to the Baffle 4 case. We observe therefore a global effect of the outer baffle quantitatively stronger than the one of the inner baffle. This is also backed up by the fact that the gas puff values for the inner and the outer baffle cases are respectively of $1.6 \cdot 10^{21} \mathrm{el} / \mathrm{s}$ and $1.8 \cdot 10^{21} \mathrm{el} / \mathrm{s}$, consistent with a lower core ionisation for the case with the outer baffle. Nevertheless, both inner and outer baffles appear to locally have an impact similar to a full set of baffles, although the inner baffle has a reduced effect on the other target. As in TCV it is generally easier to obtain detached plasma at the outer target [20], the inner baffle could, therefore, be important to access detached regimes also at the inner target. 


\section{Baffle closure scan at variable upstream condi- tions}

We focus now on the effect of the baffle closure for all the different upstream parameters listed in Table 2, and in particular for higher collisionality conditions than the cases discussed in section 3. Qualitatively, changing input power and upstream density, the baffle closure affects upstream profiles in the same way as in the formerly presented cases, with Baffle 4 or Baffle 5 generally minimising upstream neutral pressure. Since we will later show the importance of the ionisation front position in determining the performances of the baffle, it is interesting to evaluate the spatial distribution of the net ion source.
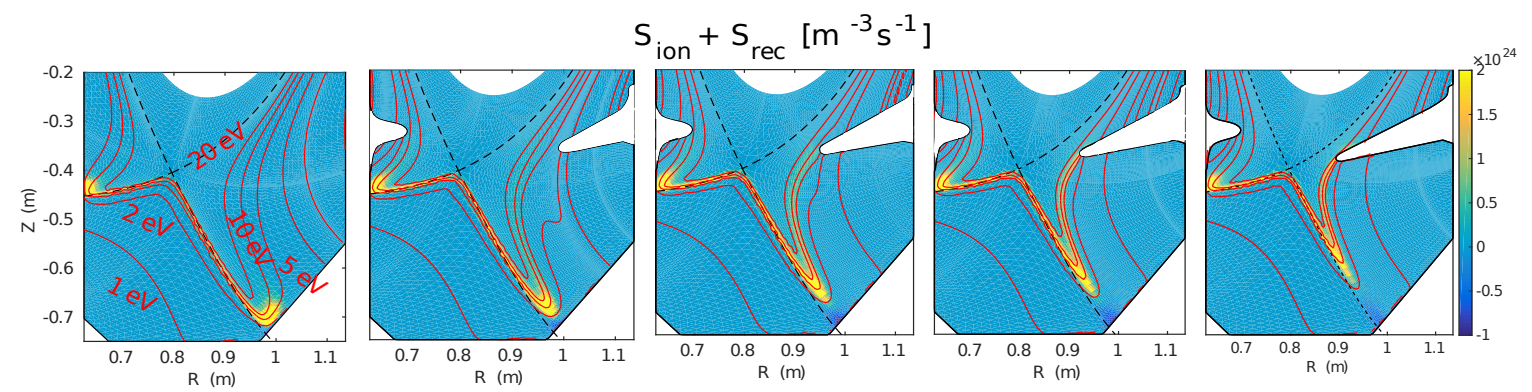

Figure 8: 2D map of the net ion source (ionisation plus recombination) in the different baffle cases, with $n_{u}=3.5 \cdot 10^{19} \mathrm{~m}^{-3}$ and $P_{\text {in }}=1.2 \mathrm{MW}$. In the first panel, contour lines indicate electron temperature levels. In the other panels, the values of the electron temperatures used for contours are the same as in the first panel.

In figure 8, the spatial distribution of the ion source is shown for higher upstream density and same input powers as the cases discussed in Sec. 3, for different baffle configurations. In the case without baffle, the ionisation source is strongly localised in the vicinity of the strike points. When increasing the baffle length, the ionisation front at the outer strike point moves towards the X-point, while recombination becomes dominant near the target. This does not necessarily mean that the plasma is completely detached from the target or that the density roll-over has occurred (see [21], Chapter 16 and references therein, for a review on this subject). Recombination starts to dominate below approximately $2 \mathrm{eV}$ in all cases (see contour lines in figure 8). The ionisation events due to particle recycling on the baffle tip accounts only for up to $\sim 3 \%$ of the total recycling flux. The ionisation source at the inner target remains attached to the target surface for all inspected baffle lengths.

Figure 9 illustrates the position of the ionisation front for the different baffle closures and all the upstream conditions listed in table 2. Here we define the ionisation front as the position along the outer divertor leg, where the sum of ionisation and recombination 
events is equal to zero.

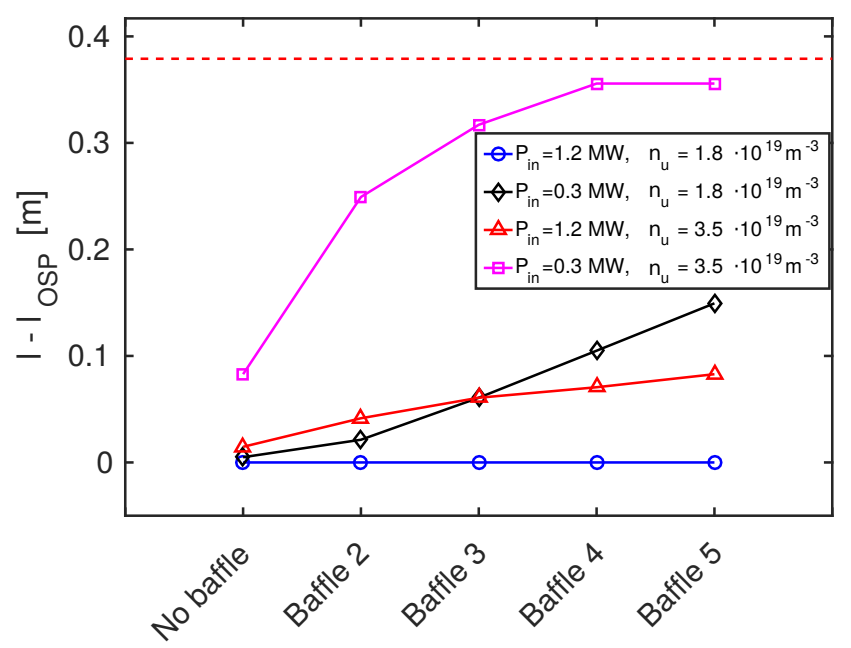

Figure 9: Ionisation front distance from the outer strike point, along the outer divertor leg, for simulations with different input power and upstream density. The red dashed line corresponds to the position of the X-point.

While in the high power, low density case discussed in section 3 , the ionisation front remains attached to the target independently of the baffle closure, in more collisional upstream conditions the ionisation front moves progressively away from the target as the baffle closure is increased. In the high density, low power case, the ionisation front reaches the X-point height, so that any further increase of the baffle length is not expected to have beneficial consequences.

The increasing poloidal distance of the ionisation front from the target is caused by the progressive cooling of the divertor plasma with an increasing outer baffle extension. Considering more specifically the high-power, high-density case, we note that the divertor dissipated power fraction (i.e., the ratio of the power dissipated in the outer divertor via photons and neutrals and the total power entering the outer divertor) increases monotonically with the outer baffle length, leading to a decrease in the peak heat flux at the outer target. This additional dissipation is mainly due to deuterium radiation and charge exchange losses. For long baffles (Baffles 4 and 5), however, large fractions of the power directed towards the outer divertor (up to $40 \%$ in the Baffle 5 case) get intercepted by the outer baffle, thus substantially contributing to the cooling and the total heat flux reduction at the outer target. 


\subsection{Effect of baffle closure on neutral compression}

In order to evaluate the compression ratio, we average the neutral density, including the molecular one as defined in section 1, over the "main chamber" and the "divertor" volumes:

$$
c_{D}=\frac{\left\langle n_{n}\right\rangle_{\text {div }}}{\left\langle n_{n}\right\rangle_{\text {main }}},
$$

where the divertor and main chamber regions are separated by a line connecting the tips of the inner and of the outer baffle. In the case without any baffle, or with just the inner baffle, a horizontal line is set as separation between the divertor and the main chamber, at the vertical position of the inner baffle tip. Note that the $c_{D}$ values calculated with (2) can be sensitive to the choice of the line separating the divertor and the main chamber regions. This is because neutral density usually drops rapidly at the X-point height. In the present simulations, however, the $c_{D}$ values are only weakly sensitive to this choice.
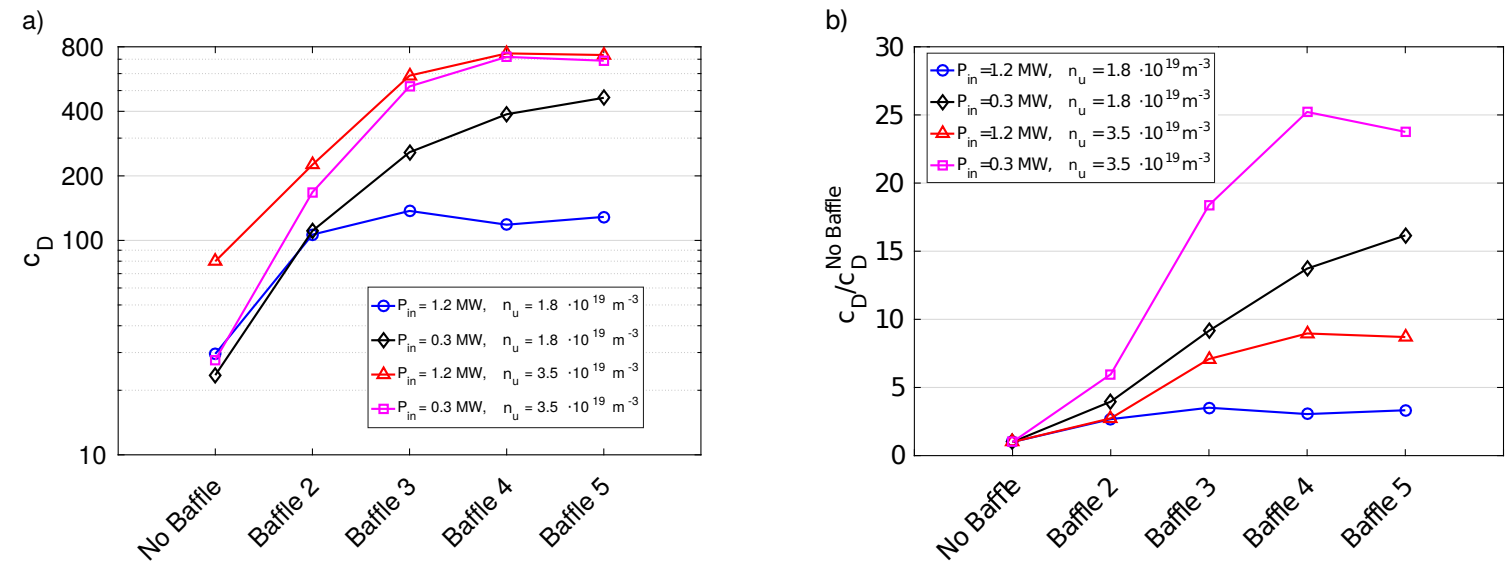

Figure 10: a) Neutral compression factor for simulations with different input power and upstream density. b) Ratio of compression factor with respect to the unbaffled case.

In the low-collisionality case described in section 3 , the neutral compression ratio is approximately 30 without baffle, and it increases by a factor of approximately 4 with Baffle 3, figure 10a, as a result of a factor 2 increase in the average neutral density in the divertor, and of a factor 2 decrease in the main chamber. This increase is slightly lower than SOLPS-ITER predictions [18]. The possibility that these quantitative differences were due the more comprehensive set of chemical reactions used in SOLPS-ITER has 
been ruled out by a dedicated set of simulations with the same reactions, which did not substantially change our results. In the Baffle 4 and 5 cases, the ion flux recycled on the LFS baffle, even if much smaller than the one at the strike points, represents the main source of particles for the main chamber, and thus affects the neutral compression ratio. We remind that in these cases, the ionisation front remains attached to the wall.

At low density and low input power, the neutral compression factor increases monotonically with the outer baffle length, figure 10a. While the ionisation front is attached in the no-baffle case, it moves towards the X-point, with increasing baffle dimensions. It is worth noting that for these conditions, while the neutral compression increases the longer the baffle, peak heat fluxes on the baffles become very high for the Baffle 4 and the Baffle 5 case ( $\sim 75 \%$ and $\sim 140 \%$ of the peak target fluxes, respectively). Increasing the upstream density at high input power, the ionisation front is still attached in the no-baffle case. In this case, the neutral compression is relatively high, due to an elevated molecule density in the Private Flux Region (PFR). These results are in line with SOLPS-ITER simulations [18], which showed an improvement in the neutral compression ratio with increasing upstream density. The best neutral compression is achieved with Baffle 4, while with Baffle 5 the recycling on the outer baffle leads to a higher ionisation source in the main chamber, and to a slightly lower $c_{D}$. In the high-density, low-power case, the baffle is particularly efficient in confining neutrals. As explained later, this is due to the fact that in these conditions the ionisation front is detached. A saturation is reached, again, for the Baffle 4 case. With Baffle 5, the ionisation front is close to the X-point, so that neutrals partially leak to the main chamber, in the SOL region between the HFS baffle and the separatrix, leading to a slightly lower neutral compression.

\subsection{Neutral density asymmetry in the divertor}

The change in neutral compression between a baffled and an unbaffled plasma appears to be larger when the ionisation front is detached. One can see that the improvement in neutral compression with respect to the unbaffled case (figure 10b) follows the same trends as the distance of the ionisation front from the outer target (figure 9). In order to understand this behaviour, we consider two cases without baffle at the same upstream density $\left(n_{u}=3.5 \cdot 10^{19} \mathrm{~m}^{-3}\right)$, but different input power $(1.2 \mathrm{MW}$ and $0.3 \mathrm{MW})$.

Figure 11a, corresponding to a high density, high power attached case, shows a difference in neutral density between the PFR and the SOL of approximately one order of magnitude. In this case, without baffles, the neutral compression is particularly elevated $\left(c_{D} \sim 80\right)$, and the PFR contains the majority of neutral particles. Figure $11 \mathrm{~b}$ shows the same case, but at low input power: here the asymmetry between SOL and PFR is much smaller. Although the ion flux to the outer target is lower than in the high power case, the neutral density in the SOL region is actually higher, and the 

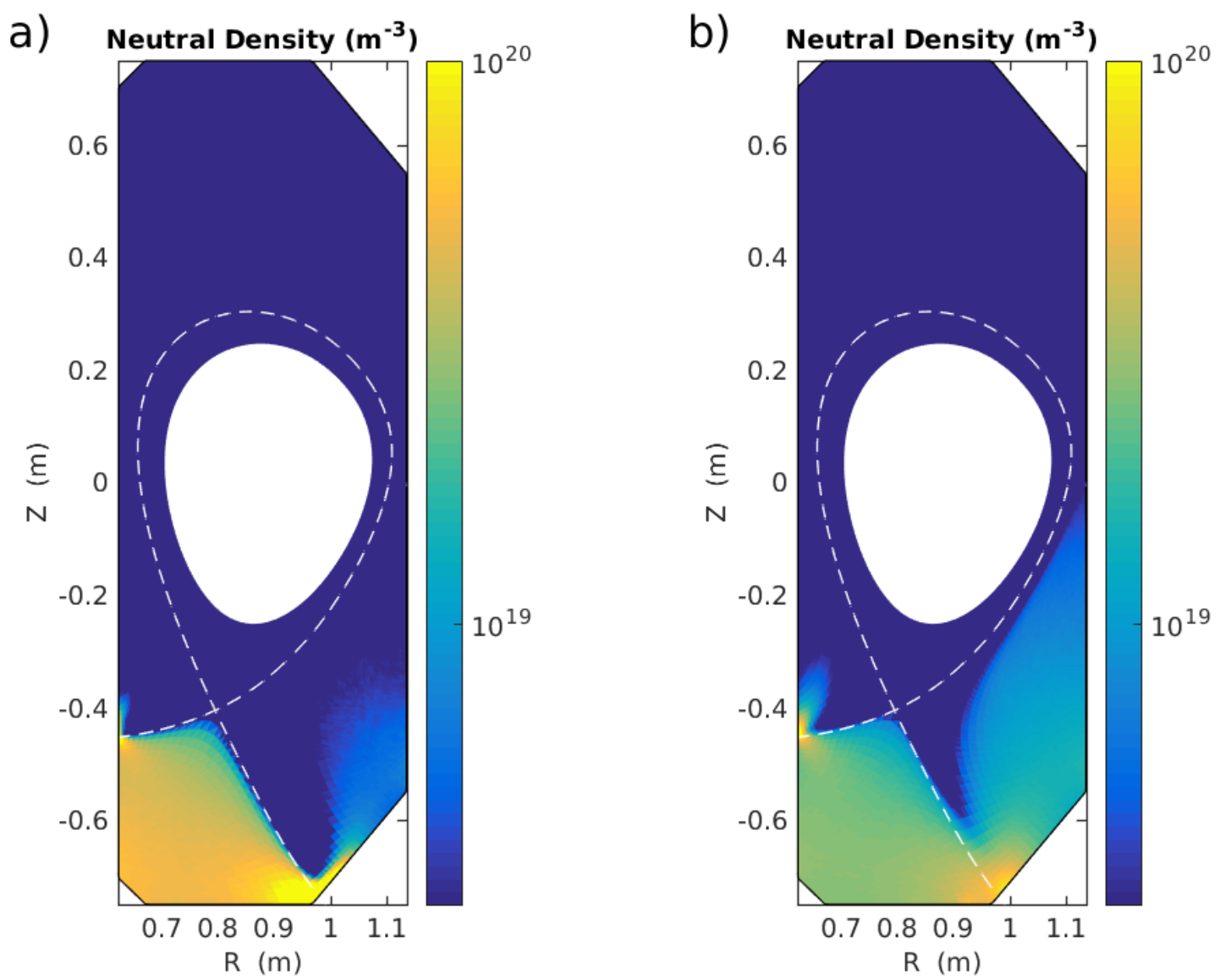

Figure 11: Neutral density in a non-baffled case at high density $\left(n_{u}=3.5 \cdot 10^{19} \mathrm{~m}^{-3}\right)$ and a) high power $(1.2 \mathrm{MW})$, and b) low power $(0.3 \mathrm{MW})$.

neutral compression factor consequently lower $\left(c_{D} \sim 30\right)$.

Since this asymmetry in neutral density affects the impact of the baffles, we need to explain its origin. The main source of neutrals is located at the targets, where impinging ions are re-emitted as "first-generation" $D_{2}$ molecules, with an energy corresponding to wall temperature (set to $310 \mathrm{~K}$ in these simulations). In the configuration investigated here, the poloidal angle between the separatrix and the wall is close to $90^{\circ}$, so the average molecule flux is directed along the separatrix direction. Therefore, the asymmetry in neutral density is not caused by the inclination of the target with respect to the separatrix (this last effect is investigated in e.g. [22], [23], [24]). The majority of recycled molecules are dissociated when they enter in a region where $T_{e}>\sim 3 \mathrm{eV}$ ([21], [25]). While the resulting ions travel back to the target, the $D^{0}$ atoms, with 
typical energies around $3 \mathrm{eV}$, can escape from the divertor leg. Subsequently, they can be reflected or absorbed by the wall, and re-emitted as molecules. The resulting slow "second-generation" molecules can travel for a relatively long time, in regions such as the PFR or the far SOL, until they get sufficiently close to the plasma to be dissociated again.

a)

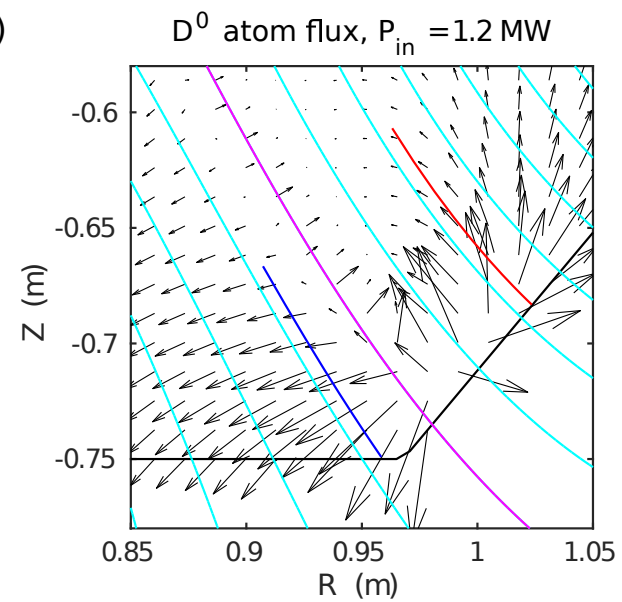

c)

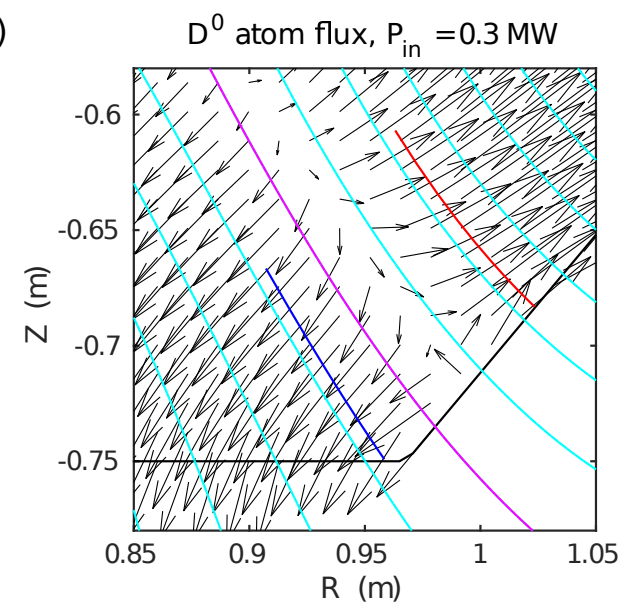

b)

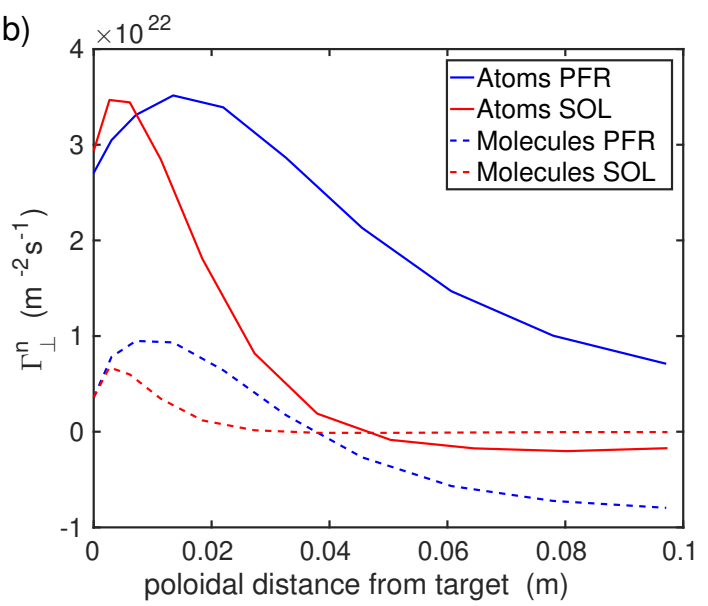

d)

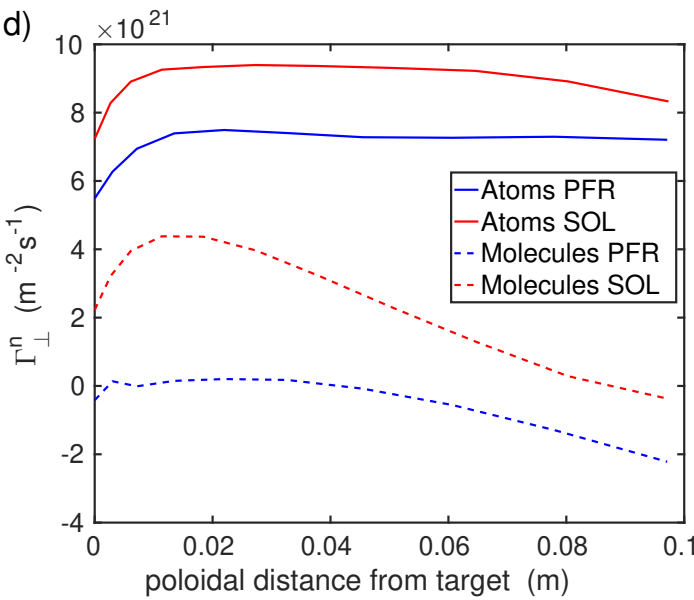

Figure 12: a): $D^{0}$ flux near the outer strike point in the case with no baffle, $n_{u}=$ $3.5 \cdot 10^{19} \mathrm{~m}^{-3}$ and $P=1.2 \mathrm{MW}$. Arrow lengths are proportional to the flux magnitude. Cyan lines represent flux surfaces, and the magenta one represents the separatrix. The blue and the red lines are the portion of flux surfaces over which the flux is evaluated. b) $D^{0}$ atom and $D_{2}$ molecule fluxes across the blue and red surfaces indicated in the a) panel. The flux is positive if directed from the separatrix towards the considered surface. c) Same plot as a), for the case with $n_{u}=3.5 \cdot 10^{19} \mathrm{~m}^{-3}$ and $P=0.3 \mathrm{MW}$. d) Same plot as b), for the case with low input power. 
In the attached case at high input power, one can see from figure 12a that the source of neutral atoms is radially located close to the separatrix, and poloidally at few centimeters from the target. This distance approximately corresponds to the mean free path for molecules dissociation (based on dissociation rate coefficients in [25]). The total atom flux in this case, which does not have, a priori, a preferential direction, is stronger towards the PFR than towards the SOL, as shown in figure 12b, where we calculate the atom and molecule fluxes across the control surfaces defined in 12a. This difference can be explained by the different ionisation mean free path of neutral atoms towards the PFR and the SOL, due to higher densities and temperatures on the SOL side of the strike point with respect to the PFR side. Figure $12 \mathrm{~b}$ also shows that the net flux of molecules (mainly "first-generation", with source centered close to the strike point) across the same surface is relatively low with respect to the atoms flux, so they are less important in determining the overall neutral densities in the PFR and in the SOL. As a result, in the attached case, neutral density is significantly higher in the PFR than in the SOL, with molecules (mainly "second-generation") accounting for approximately $90 \%$ of neutral particles in the divertor and deuterium atoms for approximately $10 \%$.

In the detached case illustrated in figure 12c, the source of neutral atoms is not peaked at the target, but extends along the entire divertor leg. The electron temperature in the SOL is insufficient to ionise the resulting neutral atoms directed towards the main SOL, similarly to the PFR. Additionally, a non-negligible net flux of molecules reaches the SOL, as shown in figure $12 \mathrm{~d}$. Globally, a higher share of neutrals, with respect to the attached case, is directed to the main chamber through the Common Flux Region, and is blocked by the baffles. In the detached case, so, baffles lead to higher changes in neutral compression ratios. Moreover, this effectiveness in reflecting neutrals increases with the baffle length. In the attached case, instead, the PFR acts as a reservoir that temporarily encloses neutrals, before they get ionised in divertor legs, so that the baffle closure plays a weaker role.

\section{Discussion: a simple evaluation of neutral flux asymmetries}

A simple model is presented here in order to evaluate the asymmetry in neutral content in the PFR and in the divertor SOL. We assume, for simplicity, that the divertor leg is perpendicular to the wall in the poloidal plane and we call $x$ the radial coordinate along the outer target. We also suppose that all the neutral atoms are generated at the position $x_{M}$ along the target, corresponding to the peak of the ion flux. Then, the ratio of the fluxes perpendicular to two surfaces placed at $x_{S O L}>x_{M}$ and $x_{P F R}<x_{M}$ 
is simply:

$$
\frac{\Gamma_{S O L}^{n}}{\Gamma_{P F R}^{n}} \simeq \frac{\exp \left(-\int_{x_{M}}^{x_{S O L}} \frac{1}{\lambda_{i}\left(n_{e}(x), T_{e}(x)\right)} d x\right)}{\exp \left(\int_{x_{M}}^{x_{P F R}} \frac{1}{\lambda_{i}\left(n_{e}(x), T_{e}(x)\right)} d x\right)}
$$

where $\lambda_{i}=v_{n} /\left(n_{e}\left\langle\sigma v_{e}\right\rangle_{i}\right)$ is the ionisation mean free path, with $v_{n}$ the atom velocity and $\left\langle\sigma v_{e}\right\rangle_{i}$ the ionisation rate coefficient. The asymmetry in radial density and temperature profiles in the PRF and the main SOL then leads to a different integrated ionisation mean free path for the two sides, which usually results in $\Gamma_{S O L}^{n} / \Gamma_{P F R}^{n} \leq 1$. In order to calculate the flux ratio, we assume that neutral atoms are originated from the dissociation of desorbed molecules, and that half of them fly towards the SOL at the thermal speed corresponding to $3 \mathrm{eV}$, while the other half is directed towards the PFR with the same velocity. As a general approximation for our plasma profiles at the outer target, we consider now a simple Eich-like profile for electron temperature, with $\lambda^{T e}=0.015 \mathrm{~m}$ and $S^{T e}=0.01 \mathrm{~m}$, and a profile of electron density with $\lambda^{n}=0.01 \mathrm{~m}$ and $S^{n}=0.006 m[26]$. These values have been found to be representative of most of the profiles resulting from our simulations. While for density this function fits well the density profiles at the target, we note that it is less so for temperature, that is difficult to fit satisfactorily with an Eich function [27]. No background value is considered for these fields. We can now multiply these density and temperature profiles by arbitrary constants, and infer the ratio in neutral flux between SOL and PRF in this ideal situation using equation (3). We fix the two boundaries of integration $x_{S O L}$ and $x_{P F R}$, where the fluxes to the SOL and to the PFR are respectively calculated, at the locations represented in figure 12a. Then, we characterise the profiles by their maximum value, and we plot the ratio of fluxes as a function of these maximum values in figure 13, in the form of contour lines. The model predicts the asymmetry to increase with the maximum temperature and density at the target, although with a weaker dependence on density in the considered parameter range.

In the same plot, we represent the same quantity, calculated for each of the SolEdge2DEIRENE simulations considered in this work. In this case, the fluxes are averaged over the poloidal extension indicated in figure 12a. The model can fail if the dissociation region of first-generation molecules is far from the target, where radial plasma profiles can differ substantially from the ones at the target. This happens for example in some high density cases, where the temperature profiles at the target is approximately flat, but it is not at the location of the atoms source. In most of the cases however, the simple model introduced here can catch qualitatively, and almost quantitatively, the imbalance in neutral fluxes directed towards PFR and SOL. This suggests that we have identified the right mechanisms that determine the asymmetry in neutral density in these attached and detached TCV simulations. The choice of atom velocity determined by molecular dissociation processes is justified for the parameter space explored 


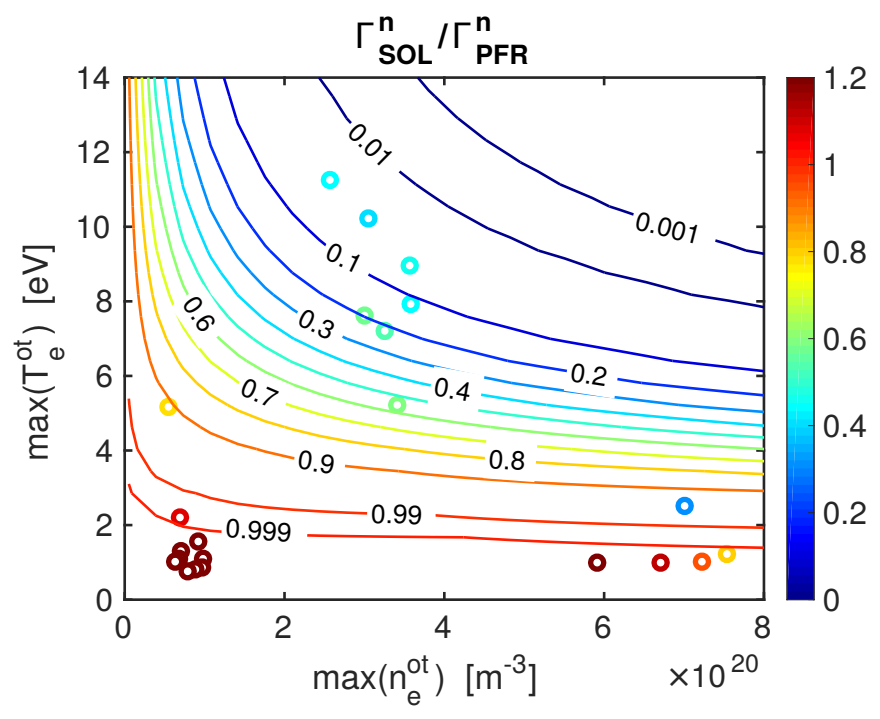

Figure 13: Ratio of the neutral atom fluxes to the Scrape-Off Layer, and to the Private Flux Region. The contour line represent the simple model described by (3), the coloured dots represent the values calculated from SolEdge2D-EIRENE simulations.

in this work and for carbon walls. In the case of a tungsten divertor, for example, a higher share of ions impinging on the target would be reflected as neutral atoms, thus leading to a higher average neutral velocity [28]. Different assumptions should therefore be made on the neutral average velocity $v_{n}$, which would change the mean free paths to be inserted in (3), but not the validity of the model, as long as the geometric hypotheses of the model hold (half of the atoms directed towards the PFR and half to the SOL at the atom source location). This simple model can be easily tested in experiments, where the ratio of neutral pressures in the SOL and in the PFR could be considered as an approximation of the ratio of the neutral fluxes to the two regions, while the temperature and density at the target can be inferred from Langmuir probes measurements.

\section{Conclusion}

The effect of the baffle closure on the TCV edge plasma has been investigated by means of 2D fluid simulations performed with the SolEdge2D-EIRENE code, taking into account the realistic geometry for plasma-facing components. A first set of simulations of low collisionality plasmas, comparable to previous SOLPS-ITER cases [10], has been analysed. Results show that an intermediate-length baffle, similar to the one installed at present on TCV, is able to optimise the neutral compression ratio, increasing it 
by at least a factor 4 with respect to the unbaffled case in these attached conditions. These results confirm previous SOLPS-ITER simulations, although the degradation of neutral compression ratio for longer outer baffles is found to be substantially weaker in SolEdge2D-EIRENE, probably because of an overestimation of the outer boundary recycling in the past work. In such low-collisionality, attached plasmas, the average neutral pressure in the divertor is not primarily determined by the outer baffle. Instead, the main reservoir of neutral particles is the PFR. The reason for this is that the $D_{2}$ molecules recycling from the target get dissociated in the divertor leg, and the resulting deuterium atoms are more likely to reach the PFR than the main SOL. As shown by a simplified analytical model, this behaviour is related to the asymmetry of the divertor radial profiles of electron density and temperature, and associated differences in the ionisation probability of the neutral atoms travelling towards the PFR and the main SOL, respectively. Therefore, as long as the divertor leg is not transparent to the deuterium molecules traveling back towards the leg after desorption from the wall, neutral particles can be well confined into the PFR.

In the same upstream conditions, the roles of the inner and of the outer baffle have been disentangled by performing two simulations where only one of the two components is present at a time. The case including only the outer baffle is quantitatively the one approaching most the performances obtained by the complete baffle. Both baffles, nevertheless, are effective in lowering locally the temperature, and thus the inner baffle is still potentially interesting for detachment studies at the inner target.

The parameter space explored by previous SOLPS baffle length scan has been extended considering multiple combinations of input power and upstream electron density, in particular considering cases at higher collisionalities. Increasing baffle closure is found to increase monotonically the ionisation front distance from the outer strike point, allowing recycled neutral atoms to freely fly to the SOL and then to the main chamber. As a result, the efficiency of the baffle in inhibiting the movement of neutrals to the main chamber is improved with respect to attached cases, leading to compression factors up to 25 times higher than unbaffled cases. For high densities, the optimum compression ratio is obtained with Baffle 4, while for Baffle 5, depending on the level of input power, either a significative recycling occurs on the baffle tip, or the ionisation front moves too close to the X-point, reducing plasma plugging and allowing neutrals to escape from the divertor region. A key observation is also that in some cases (low density, low power), even though an increase in the outer baffle length can still lead to an improved compression ratio, the peak heat flux on the baffle tip becomes a large fraction of the peak target flux, which would be a concern for a reactor. A baffle length corresponding to the presented Baffle 4 scenario may lead to increased neutral compression ratios with respect to the ones obtained with the present baffle, and this without an excessive heating of the outer baffle.

While in this work we have considered a purely diffusive perpendicular transport, sim- 
ulations including drifts will be carried out in a near future. Drifts are expected to lead to a different imbalance between inner and outer divertor (see e.g. [29]), depending on toroidal field direction, and possibly cause an additional neutral influx into the confined region [30]. The results of more complex simulations will be interpreted also following the guidelines given by this work, where we have highlighted how the target conditions and the ionisation source distribution can affect the neutral compression ratio for a given baffle geometry. A more precise description of cross-field transport in the far SOL could also quantitatively impact the particle recycling on the baffles. This calls for turbulent simulations including neutral dynamics and a realistic wall geometry.

\section{Acknowledgements}

This work has been carried out within the framework of the EUROfusion Consortium and has received funding from the Euratom research and training programme 2014-2018 and 2019-2020 under grant agreement No 633053. The views and opinions expressed herein do not necessarily reflect those of the European Commission. This work was supported in part by the Swiss National Science Foundation. This work was performed within the 3rd cycle of MARCONI-FUSION HPC. The required computational resources were allocated under the project PEXUPG.

\section{References}

[1] A W Leonard. Plasma detachment in divertor tokamaks. Plasma Physics and Controlled Fusion, 60(4):044001, feb 2018.

[2] A. Loarte. Effects of divertor geometry on tokamak plasmas. Plasma Physics and Controlled Fusion, 43(6):R183-R224, 2001.

[3] M. V. Umansky, B. LaBombard, D. Brunner, M. E. Rensink, T. D. Rognlien, J. L. Terry, and D. G. Whyte. Attainment of a stable, fully detached plasma state in innovative divertor configurations. Physics of Plasmas, 24(5):056112, 2017.

[4] E Havlíčková, J Harrison, B Lipschultz, G Fishpool, A Kirk, A Thornton, M Wischmeier, S Elmore, and S Allan. SOLPS analysis of the MAST-u divertor with the effect of heating power and pumping on the access to detachment in the super-x configuration. Plasma Physics and Controlled Fusion, 57(11):115001, aug 2015.

[5] H.Y. Guo, C.F. Sang, P.C. Stangeby, L.L. Lao, T.S. Taylor, and D.M. Thomas. Small angle slot divertor concept for long pulse advanced tokamaks. Nuclear Fusion, 57(4):044001, feb 2017. 
[6] X. J. Liu, L. Wang, G. Z. Deng, J. C. Xu, J. B. Liu, S. L. Gao, C. R. Wu, J. Huang, L. Zhang, G. Q. Li, and X. Gao. Modeling study of the onset density for divertor detachment on east. Physics of Plasmas, 26(10):102510, 2019.

[7] S. Coda et al. Physics research on the TCV tokamak facility: from conventional to alternative scenarios and beyond. Nuclear Fusion, 59(11):112023, aug 2019.

[8] A. Fasoli and. TCV heating and in-vessel upgrades for addressing DEMO physics issues. Nuclear Fusion, 55(4):043006, mar 2015.

[9] H. Reimerdes, S. Alberti, P. Blanchard, P. Bruzzone, R. Chavan, S. Coda, B.P. Duval, A. Fasoli, B. Labit, B. Lipschultz, T. Lunt, Y. Martin, J.-M. Moret, U. Sheikh, B. Sudki, D. Testa, C. Theiler, M. Toussaint, D. Uglietti, N. Vianello, and M. Wischmeier. Tcv divertor upgrade for alternative magnetic configurations. Nuclear Materials and Energy, 12:1106 - 1111, 2017. Proceedings of the 22nd International Conference on Plasma Surface Interactions 2016, 22nd PSI.

[10] A. Fasoli, H. Reimerdes, S. Alberti, M. Baquero-Ruiz, B.P. Duval, E. Havlikova, A. Karpushov, J.-M. Moret, M. Toussaint, H. Elaian, M. Silva, C. Theiler, and D. Vaccaro and. TCV heating and divertor upgrades. Nuclear Fusion, 60(1):016019, nov 2019.

[11] S. Wiesen, D. Reiter, V. Kotov, M. Baelmans, W. Dekeyser, A.S. Kukushkin, S.W. Lisgo, R.A. Pitts, V. Rozhansky, G. Saibene, I. Veselova, and S. Voskoboynikov. The new solps-iter code package. Journal of Nuclear Materials, 463(Supplement C):480 - 484, 2015. PLASMA-SURFACE INTERACTIONS 21.

[12] X. Bonnin, W. Dekeyser, R. Pitts, D. Coster, S. Voskoboynikov, and S. Wiesen. Presentation of the new solps-iter code package for tokamak plasma edge modelling. Plasma and Fusion Research, 11:1403102, 2016.

[13] D. Reiter. Progress in two-dimensional plasma edge modelling. Journal of Nuclear Materials, 196-198(Supplement C):80 - 89, 1992. Plasma-Surface Interactions in Controlled Fusion Devices.

[14] H. Bufferand, G. Ciraolo, Y. Marandet, J. Bucalossi, Ph. Ghendrih, J. Gunn, N. Mellet, P. Tamain, R. Leybros, N. Fedorczak, F. Schwander, and E. Serre. Numerical modelling for divertor design of the west device with a focus on plasmawall interactions. Nuclear Fusion, 55(5):053025, 2015.

[15] A. Paredes, H. Bufferand, G. Ciraolo, F. Schwander, E. Serre, P. Ghendrih, and P. Tamain. A penalization technique to model plasma facing components in a tokamak with temperature variations. Journal of Computational Physics, 274:283 - 298, 2014. 
[16] J. Bohdansky. A universal relation for the sputtering yield of monatomic solids at normal ion incidence. Nuclear Instruments and Methods in Physics Research Section B: Beam Interactions with Materials and Atoms, 2(1):587 - 591, 1984.

[17] L. Casali, C. Sang, A.L. Moser, B.M. Covele, H.Y. Guo, and C. Samuell. Modelling the effect of divertor closure on detachment onset in diii-d with the solps code. Contributions to Plasma Physics, 58(6-8):725-731, 2018.

[18] M Wensing, B P Duval, O Février, A Fil, D Galassi, E Havlickova, A Perek, H Reimerdes, C Theiler, K Verhaegh, M Wischmeier, and and. SOLPS-ITER simulations of the TCV divertor upgrade. Plasma Physics and Controlled Fusion, 61(8):085029, jul 2019.

[19] N. Vianello, C. Tsui, C. Theiler, S. Allan, J. Boedo, B. Labit, H. Reimerdes, K. Verhaegh, W.A.J. Vijvers, N. Walkden, S. Costea, J. Kovacic, C. Ionita, V. Naulin, A.H. Nielsen, J. Juul Rasmussen, B. Schneider, R. Schrittwieser, M. Spolaore, D. Carralero, J. Madsen, B. Lipschultz, F. Militello, and and. Modification of SOL profiles and fluctuations with line-average density and divertor flux expansion in TCV. Nuclear Fusion, 57(11):116014, aug 2017.

[20] C. Theiler, B. Lipschultz, J. Harrison, B. Labit, H. Reimerdes, C. Tsui, W.A.J. Vijvers, J. A. Boedo, B.P. Duval, S. Elmore, P. Innocente, U. Kruezi, T. Lunt, R. Maurizio, F. Nespoli, U. Sheikh, A.J. Thornton, S.H.M. van Limpt, K. Verhaegh, and N. Vianello and. Results from recent detachment experiments in alternative divertor configurations on TCV. Nuclear Fusion, 57(7):072008, mar 2017.

[21] P. C. Stangeby. The Plasma Boundary of Magnetic Fusion Devices. Bristol: Institute of Physics Publishing, 2000.

[22] Chaofeng Sang, H.Y. Guo, P.C. Stangeby, L.L. Lao, and T.S. Taylor. SOLPS analysis of neutral baffling for the design of a new diverter in DIII-d. Nuclear Fusion, 57(5):056043, apr 2017.

[23] D. Moulton, G. Corrigan, J.R. Harrison, and B. Lipschultz and. Neutral pathways and heat flux widths in vertical- and horizontal-target EDGE2d-EIRENE simulations of JET. Nuclear Fusion, 58(9):096029, jul 2018.

[24] A Fil, B Lipschultz, D Moulton, B D Dudson, O Février, O Myatra, C Theiler, $\mathrm{K}$ Verhaegh, and M Wensing. Separating the roles of magnetic topology and neutral trapping in modifying the detachment threshold for TCV. Plasma Physics and Controlled Fusion, 62(3):035008, jan 2020. 
[25] D.E. Post, D.B. Heifetz, and M. Petravic. Models for poloidal divertors. Journal of Nuclear Materials, 111-112:383 - 395, 1982.

[26] T. Eich, B. Sieglin, A. Scarabosio, W. Fundamenski, R. J. Goldston, and A. Herrmann. Inter-elm power decay length for jet and asdex upgrade: Measurement and comparison with heuristic drift-based model. Phys. Rev. Lett., 107:215001, Nov 2011.

[27] F. Wagner. A study of the perpendicular particle transport properties in the scrape-off layer of asdex. Nuclear Fusion, 25(5):525, 1985.

[28] Chen Zhang, Chaofeng Sang, Liang Wang, Mingyu Chang, Daoyuan Liu, and Dezhen Wang. Effect of carbon and tungsten plasma-facing materials on the divertor and pedestal plasma in EAST. Plasma Physics and Controlled Fusion, 61(11):115013, oct 2019 .

[29] N Christen, C Theiler, TD Rognlien, ME Rensink, H Reimerdes, R Maurizio, and B Labit. Exploring drift effects in TCV single-null plasmas with the UEDGE code. Plasma Physics and Controlled Fusion, 59(10):105004, aug 2017.

[30] F. Reimold, M. Wischmeier, S. Potzel, L. Guimarais, D. Reiter, M. Bernert, M. Dunne, and T. Lunt. The high field side high density region in solps modeling of nitrogen-seeded h-modes in asdex upgrade. Nuclear Materials and Energy, $12: 193-199,2017$. 